\title{
Evaluation of Dry Eye after Implantable Collamer Lens Surgery
}

\author{
Haiting Chen Xueyan Feng Guangzeng Niu Yuxiang Fan \\ Department of Ophthalmology, Cangzhou Central Hospital, Cangzhou, China
}

\section{Keywords}

Implantable Collamer Lens · Dry eye · Meibomian gland dysfunction

\begin{abstract}
Objectives: To evaluate dry eye (DE) and associated meibomian gland dysfunction parameters after Implantable Collamer Lens (ICL) surgery. Methods: This is a prospective observational case series. Patients who underwent ICL implantation without previous ocular diseases or ophthalmic treatments were enrolled. Their Ocular Surface Disease Index (OSDI), noninvasive breakup time (NIBUT), meibography, slit-lamp examination of the lid margin, corneal fluorescein staining (CFS), and Schirmer test I were examined preoperatively and at 1 and 3 months postoperatively. Results: A total of 117 eyes of 60 patients were enrolled, and 107 eyes completed 3-month follow-up period. OSDI, lid marginal abnormality, and meibomian gland (MG) secretion, and meibum quality score were significantly higher at 1 month postoperatively and recovered partially at 3 months after surgeries, while NIBUT was significantly decreased all the time. Patients with previous DE symptoms (OSDI score $\geq 12$ ) showed not only lower Schirmer and TBUT values but also higher CFS, lid margin score, MG loss, MG secretion, and meibum quality scores compared with those in the control
\end{abstract}

group after operations. Low Schirmer, NIBUT values, and high meibum quality score were determined as risk factors for DE symptoms after ICL surgery. Conclusions: ICL implantation has a bad influence on the ocular surface and MG functions. The influence may be more obvious in patients with existing DE.

C 2020 S. Karger AG, Basel

\section{Introduction}

The Visian Implantable Collamer Lens (ICL; STAAR Surgical Co., CA, USA) are a kind of posterior chamber phakic intraocular lens, which have been proved to be effective, predictable, stable, and safe for the correction of ametropia $[1,2]$. Recently, a growing amount of refractive surgeons and patients have selected ICL implantation because of its reversibility, broader range of ametropia correction, and good visual outcomes [3-5].

However, dry eye (DE), which was due to tear film dysfunction, is just one of the most frequent complications after ICL surgery. Many patients experience DE symptoms, such as foreign body sensations, dryness, irritation, intermittent pain, ocular fatigue, transient blurred vision, and red eye [6]. Although in most of the patients DE is temporary, some patients complain about severe symp- 
toms even for months after successful surgeries. This side effect influences their satisfaction and causes frustration in both patients and surgeons.

It is well known that the postoperative DE often occurs after cataract, photorefractive keratectomy, or strabismus surgeries $[7,8]$. The exact mechanism remains equivocal to date, but it is deemed to be multifactorial. Possible mechanisms include surgical incision, goblet cell damage, inflammation, and toxic effects of topical drops [9]. Recently, researchers have found that meibomian gland dysfunction (MGD) also plays an important role in postoperative DE [10]. It is not clear whether the same mechanism works after ocular ICL surgeries. There have been few studies focused on this, and our current study is aimed to assess the perioperative parameters that may cause DE syndromes, to identify risk factors, and to minimize patients' discomforts.

\section{Materials and Methods}

\section{Population Enrollment}

This prospective observational trial was performed in the Ophthalmological Institute of Cangzhou Central Hospital (Hebei, China). The study was approved by the Cangzhou Central Hospital Ethics Committee and registered at the Chinese Clinical Trial Registry (ChiCTR-INR-16008896). We enrolled patients who accepted the implantation of ICL from January 2018 to June 2019. Patients were selected following these inclusion criteria: (1) age of at least 18 years with stable refraction for 2 years, (2) soft contact lenses have been removed for at least 2 weeks and rigid contact lenses have been removed for at least 1 month before the examination, and (3) no drugs affecting tear secretion and stability were used. The exclusion criteria were (1) known ocular diseases such as corneal opacity, glaucoma, cataract, uveitis, retinal detachment, or a history of previous trauma or other ocular surgeries in 1 year; (2) presence of severe surgical complications such as ICL rotation; and (3) patients who disagreed to participate in this study or had poor compliance. These protocols followed the tenets of the Declaration of Helsinki, and written informed consent was obtained from all patients after they received a full explanation of the nature and possible consequences of the study.

\section{Patient Evaluation}

All patients underwent a series of ocular surface examinations as follows: Ocular Surface Disease Index (OSDI), the noninvasive breakup time (NIBUT), meibography, slit-lamp examination of the lid margin, corneal fluorescein staining (CFS), a 30-min break, and Schirmer test I (STI) at last. OSDI questionnaire was completed for each patient in order to assess subjective symptoms [11]. The result was calculated as a score between 0 and 100, and patients who got 13 or greater were considered symptomatic. NIBUT was measured by a Keratograph 5M (OCULUS, Germany). Patients were instructed to open eyes persistently for $17 \mathrm{~s}$ and the mean values of 3 measurements were used for analyses [7].
Evaluation of Meibomian Gland Function

Lid margin abnormalities were scored from 0 to 4 grades according to the presence of vascular engorgement, gland orifice obstruction, lid margin irregularity, and mucocutaneous junction displacement [12]. Furthermore, each eyelid was ectropionized and evaluated by a Keratograph 5M (OCULUS, Germany) to quantitate the atrophy and obstruction of meibomian glands (MGs). The extent of MG loss was scored as follows (meibography score): 0 , no absence; $1,<1 / 3 ; 2$, between $1 / 3$ and $2 / 3$; and $3,>2 / 3$. The sum of the upper and lower lids scores was recorded as the total $M G$ loss score ranging from 0 to 6 [13]. Moreover, the MG secretion was measured using a MG evaluator (Tear Science, Inc., Morrisville, NC, USA). Five glands from the center of upper eyelids were extruded with stable pressure and assessed as follows: - score 0: secretion was seen in all 5 MGs; score 1: 3-4 glands; score 2: 1-2 glands; and score 3, none of the 5 glands [14]. The quality of meibum was evaluated as follows: 0 , clear liquid secretion; 1 , cloudy liquid secretion; 2, inspissated and granular in liquid; and 3, toothpaste-like secretion [9].

CFS was performed by placing a fluorescein strip into the lower conjunctival fornix, then the amounts of fluorescein staining were examined under blue light illumination and graded using the Oxford grading scheme [15]: staining of 3 zones of the ocular surface (cornea and nasal and temporal conjunctiva) was graded from 0 to 5 in order of increasing severity, and the total maximum score was 15 points.

The Schirmer tests were performed with sterile strips (Eagle Vision, Memphis, TN, USA) inserted into the mid-lateral conjunctival sac without anesthesia, and the patients were asked to close eyes for $5 \mathrm{~min}$ in a dark room under constant temperature and humidity [7]. The lengths of wet strips were measured and recorded by the same clinician. All the patients were examined preoperatively and at 1 and 3 months postoperatively, and the assessments were made by the same physicians.

\section{Surgical Procedures}

All the surgeries were completed by the same experienced surgeon (F.Y.). Pupils were dilated with propanamide phenylephrine eye drops (Santen Pharmaceutical Co., Ltd, Osaka, Japan) 30 min before surgery. Under topical anesthesia, superior or inferior corneal paracentesis incision was performed, and ICL was inserted and placed in the posterior chamber. Finally, the anterior chamber was washed and maintained with balanced salt solutions. After surgery, antibiotics ( $0.5 \%$ levofloxacin eye drops; Santen Pharmaceutical Co.) and steroidal (tobramycin and dexamethasone eye drops; Alcon NV, TX, USA) drugs were administered topically 4 times daily for 7 days and then gradually reduced.

\section{Statistical Analysis}

Data were analyzed using SPSS software (version 19.0; SPSS, Inc., Chicago, IL, USA). The results are expressed as mean \pm standard deviation, and the normality of samples was checked by the Kolmogorov-Smirnov test. A linear mixed model with Bonferroni post hoc analysis was used to examine repeated measurements of continuous variables. A generalized linear mixed model with Bonferroni post hoc analysis was used to evaluate repeated measurements of noncontinuous variables. Between 2 groups, the Student's $t$ test was used to assess the differences if the data were normally distributed, while the Mann-Whitney test was used for the data that were not normally distributed. A $\chi^{2}$ test was used to ana- 
Table 1. Outcomes of ocular surface and MG parameters preoperation and 1 and 3 months postoperation

\begin{tabular}{|c|c|c|c|c|c|c|}
\hline \multirow[t]{2}{*}{ Variables } & \multicolumn{3}{|l|}{ Mean \pm SD } & \multicolumn{3}{|l|}{$p$ value } \\
\hline & pre-op & $\begin{array}{l}1 \text { month } \\
\text { post-op }\end{array}$ & $\begin{array}{l}3 \text { months } \\
\text { post-op }\end{array}$ & $\begin{array}{l}\text { pre-op versus } \\
1 \text { month post-op }\end{array}$ & $\begin{array}{l}\text { pre-op versus } \\
3 \text { months post-op }\end{array}$ & $\begin{array}{l}1 \text { month versus } \\
3 \text { months post-op }\end{array}$ \\
\hline OSDI & $10.79 \pm 8.18$ & $17.78 \pm 13.74$ & $11.80 \pm 7.51$ & $0.000^{* *}$ & 1.000 & $0.000^{* *}$ \\
\hline Schirmer, mm & $9.56 \pm 4.61$ & $8.36 \pm 4.11$ & $9.64 \pm 3.82$ & 0.109 & 1.000 & 0.075 \\
\hline NIBUT, s & $10.01 \pm 3.53$ & $3.66 \pm 2.21$ & $8.47 \pm 2.62$ & $0.000^{* *}$ & $0.000^{* *}$ & $0.000^{* *}$ \\
\hline CFS & $0.10 \pm 0.31$ & $0.22 \pm 0.42$ & $0.21 \pm 0.41$ & 0.062 & 0.098 & 1.000 \\
\hline Lid margin & $0.34 \pm 0.60$ & $0.85 \pm 0.88$ & $0.57 \pm 0.65$ & $0.000^{* *}$ & 0.053 & $0.014^{*}$ \\
\hline MG loss & $0.40 \pm 0.67$ & $0.49 \pm 0.74$ & $0.42 \pm 0.66$ & 1.000 & 1.000 & 1.000 \\
\hline MG secretion & $0.33 \pm 0.58$ & $0.84 \pm 0.88$ & $0.54 \pm 0.59$ & $0.000^{* *}$ & 0.074 & $0.006^{*}$ \\
\hline Meibum quality & $0.43 \pm 0.60$ & $0.77 \pm .081$ & $0.65 \pm 0.69$ & $0.002 *$ & 0.061 & 0.734 \\
\hline
\end{tabular}

Continuous variables were analyzed by linear mixed model with Bonferroni post hoc analysis. Noncontinuous variables were analyzed by generalized linear mixed model with Bonferroni post hoc analysis. MG, meibomian gland; pre-op, preoperation; post-op, postoperation; OSDI, Ocular Surface Disease Index; NIBUT, noninvasive breakup time; CFS, corneal fluorescein staining. ${ }^{*} p<0.05$. ${ }^{* *} p<0.001$.

lyze the enumerative data. The correlations between various factors and DE symptoms were studied using multivariate logistic regression analysis. A value of $p<0.05$ was considered statistically significant.

\section{Results}

\section{Demographics}

117 eyes of 60 patients ( 32 females and 28 males; mean age: $26.3 \pm 8.7$ years) were enrolled in the study. Five patients (10 eyes) were lost to follow-up and 55 patients (107 eyes) completed all examinations at 3 months postoperatively (31 females and 24 males; mean age: $25.1 \pm 4.1$ years).

\section{Changes in DE and MGD Parameters before and after ICL Surgeries}

The characteristics of all the enrolled eyes before as well as 1 and 3 months after operations are shown in Table 1. OSDI, lid marginal abnormality, and MG secretion were significantly higher than the preoperative baselines at 1 month postoperatively (all $p<0.001$, respectively; Table 1), and these parameters returned to the preoperative level at 3 months postoperatively $(p=1.000, p=0.053$, $p=0.074$, respectively; Table 1$)$. Furthermore, compared to the preoperative level, the meibum quality score increased moderately at 1 month postoperative $(p=0.002)$ and decreased mildly at a 3-month time point $(p=0.734$ between 1 month postoperative vs. 3 months postoperative). Moreover, the NIBUT was significantly decreased at 1 and 3 months postoperatively. The latter values were much higher than the former but still not recovered to preoperative levels $(p<0.001$ between each time point, respectively; Table 1). However, the Schirmer test score, CFS, and MG loss scores showed no significant changes at all times.

\section{DE Symptoms}

Patients were divided into 2 groups according to OSDI score: patients with previous DE symptoms (OSDI score $>13$ ) as DE group and patients without previous DE symptoms (OSDI score $\leq 12$ ) as a control group. The former group included 40 eyes (20 individuals, 11 females and 9 males; mean age: $25.7 \pm 4.4$ years) and the latter group involved 67 eyes ( 35 individuals, 20 females and 15 males; mean age: $24.8 \pm 4.0$ years). There were no significant differences in age ( $p=0.282$, Student's $t$ test) or gender distribution ( $p=0.147, \chi^{2}$ test $)$.

The preoperative and postoperative ocular surface parameters of both groups are presented in Figure 1. Before operation, patients in DE group showed lower Schirmer values $(p=0.000)$ and NIBUT $(p=0.000)$, and higher MG loss $(p=0.007)$, MG secretion $(p=0.016)$, and meibum quality scores $(p=0.023)$ compared with those in control group. At 1 month after operation, patients in DE group showed not only decreased Schirmer $(p=0.000)$ and NIBUT $(p=0.000)$ values and increased MG loss $(p=$ $0.004)$, MG secretion $(p=0.002)$, and meibum quality scores $(p=0.000)$, but also increased lid margin scores $(p=0.001)$ compared with those in control group. Moreover, at 3 months after operations, all the parameters dif- 


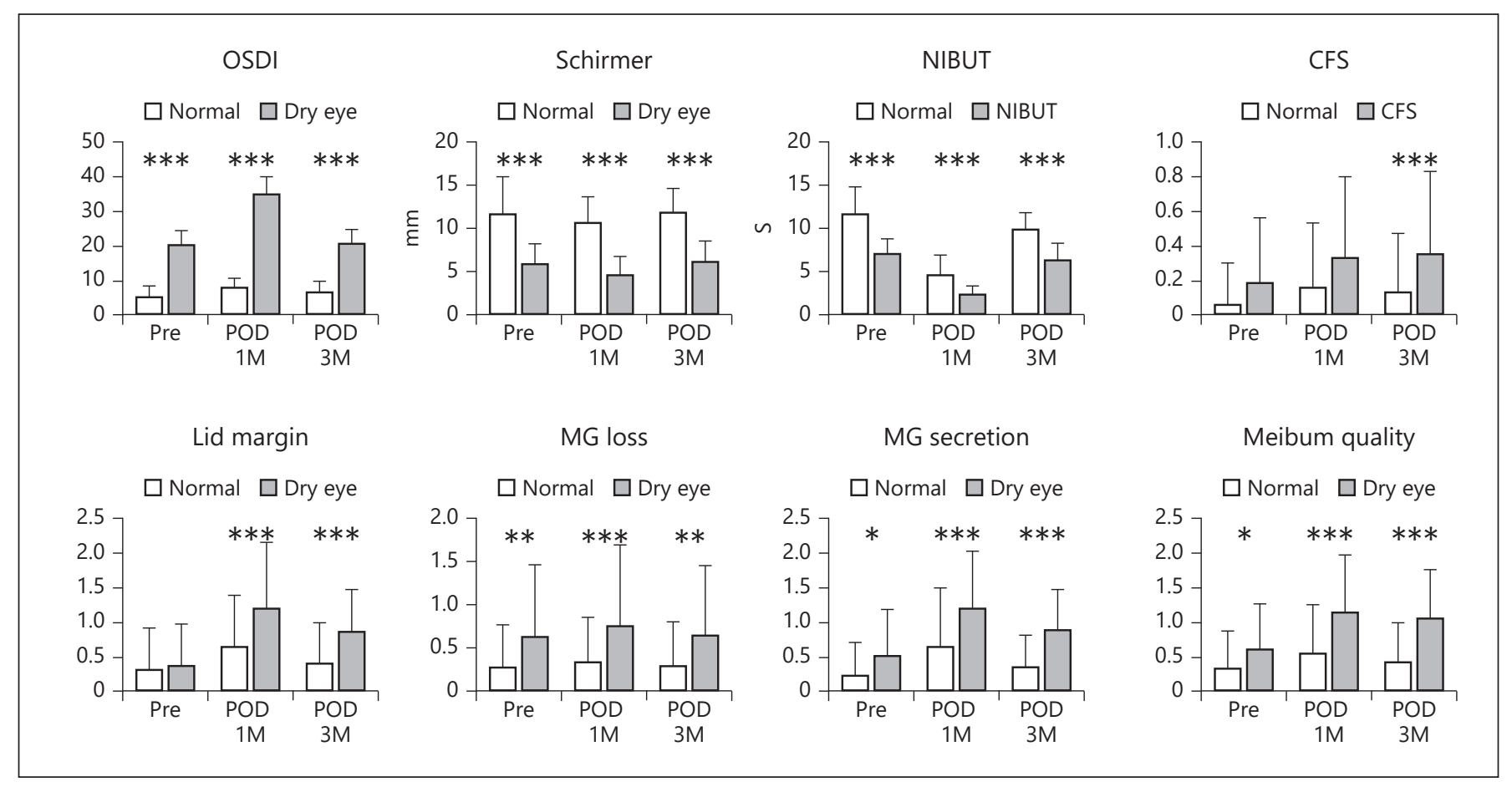

Fig. 1. Comparison of ocular surface and MG parameters between 2 groups at 3 investigated points (preoperation and 1 and 3 months postoperation) $\left({ }^{* * *} p<0.001,{ }^{* *} p<0.01,{ }^{*} p<0.05\right)$. MG, meibomian gland; OSDI, Ocular Surface Disease Index; NIBUT, noninvasive breakup time; CFS, corneal fluorescein staining.

Table 2. Correlations of DE parameters and MGD parameters with previous DE symptoms

\begin{tabular}{llllllrlrr}
\hline Variables & Gender & Age & $\begin{array}{l}\text { Schirmer, } \\
\text { mm }\end{array}$ & NIBUT, s & CFS & Lid margin & MG loss & $\begin{array}{l}\text { MG } \\
\text { secretion }\end{array}$ & $\begin{array}{l}\text { Meibum } \\
\text { quality }\end{array}$ \\
\hline$T$ value & 0.022 & 0.594 & -4.513 & -4.003 & -0.782 & -1.941 & 1.041 & -0.819 \\
$p$ value & 0.983 & 0.554 & $0.000^{* *}$ & $0.000^{* *}$ & 0.436 & 0.055 & 0.300 & 0.415 & $\begin{array}{l}2.407 \\
0.018^{*}\end{array}$ \\
\hline
\end{tabular}

The correlations between various factors were studied using Pearson's correlation coefficient. DE, dry eye; MGD, meibomian gland dysfunction; NIBUT, noninvasive breakup time; CFS, corneal fluorescein staining; MG, meibomian gland. ${ }^{*} p<0.05 .{ }^{* *} p<0.001$.

fered statistically between the 2 groups (all $p<0.01$ ). In other words, patients with previous DE symptoms had more severe DE and MGD scores at 1 and 3 months after surgery.

\section{Risk Factors for Persistent and Severe DE}

As shown in Table 2, the scores of Schirmer, NIBUT, CFS, lid margin, MG loss, MG secretion, and meibum quality were analyzed for assessing risk factors for significant and prolonged DE symptoms after ICL surgery. Schirmer, NIBUT, and meibum quality scores were sta-

Dry Eye after ICL Implantation tistically significant. The changes in meibum quality scores were positively correlated with the change in OSDI, while the changes in NIBUT and Schirmer scores showed a significant negative correlation.

\section{Discussion}

DE symptoms are frequent complaints due to tear film dysfunction, such as burning pain, irritation, fatigue, and ocular blurry. They affect not only patients' daily work 
and living but also mental health. Many patients may feel uncomfortable and dissatisfied after ocular operations because of DE syndrome. Recently, we found similar problems in patients who underwent ICL implantation operation. Therefore, in this study, we analyzed the parameters of DE and ocular surface before and after ICL surgery to identify related factors for early prediction and intervention.

As for DE parameters, OSDI scores increased, while NIBUT decreased significantly at 1 month postoperatively, which restored slowly and did not return to the original level at 3 months after surgeries. As regards MG parameters, lid marginal abnormality and $\mathrm{MG}$ secretion were significantly higher at 1 month postoperatively and returned near the baselines gradually at 3 months postoperatively. According to previous research results, the analogous situation occurred after anterior segment surgeries for the following reasons. First, the incision injury: nerve fibers were damaged by incision, leading to decreased corneal sensation and nutritional factors. Second, drug: perioperative application of antibiotics and anesthetic drugs may produce toxic effects. Third, operation stimulation: operation mechanical damage and postoperative inflammation could cause cell apoptosis or dysfunction, thereby reducing tear film stability [16].

As we know, the ICL implantation has the same incision as cataract ultrasonic emulsification surgery and similar perioperative medication, and DE is also one of the most frequent complaints after cataract surgery. Kasetsuwan et al. [17] reported a high incidence of DE (9.8\%) after phacoemulsification. Symptoms and signs of DE occur as early as 7 days postoperation and improved gradually within 3 months. The preoperative and 1 and 3 months postoperative mean OSDI scores were 12.57, 17.34 , and 16.88 , respectively. NIBUT $(12.15,5.11$, and $5.21 \mathrm{~s}$ ), Oxford schema (grades 1, 1, and 0), and STI without anesthesia $(14.14,9.83$, and 10.25) also showed a trend toward DE syndrome. It was worse according to Iglesias E's research that DE-like symptoms were reported in $32 \%$ of individuals even 6 months after cataract surgery [8]. Li et al. [18] found the incidence of DE increased dramatically after cataract surgery according to rising OSDI scores. The lacrimal river line became narrow, BUT and STI decreased, and serious squamous metaplasia occurred in the epithelial layer of the globe conjunctiva. Choi et al. [19] reported 31 of 96 patients had persistent DE symptoms at 3 months postoperatively. They discovered decreased TBUT scores as well as the lid margin abnormality, meibum quality, and MGD stage changes. These changes and trends are comparable to our results.
However, our parameters recovered quickly at 3 months postoperatively compared with those in the above investigations, perhaps because our study subjects were younger, healthier, and having stronger recovery ability.

The demographics of patients who underwent refractive surgeries resembled those of our study subjects. Li et al. [20] assessed the clinical data changes of Chinese young people (mean age, 28.21 years) before and after small incision lenticule extraction (SMILE). The preoperative OSDI was 12.26 . The numbers turned to 16.72 at 1 month and 14.91 at 3 months after surgeries. Not coincidentally, in the study of Xia et al. [21] (mean age, 25.15 years), the OSDI was 10.74 preoperatively and increased to 20.34 one month postoperatively and returned to 14.91 three months postoperatively. These quantitative data and change trends are similar to the results of our research. Furthermore, Li et al. [20] reported the BUT values were $8.58,5.68$, and $5.03 \mathrm{~s}$ before operation and 1 and 3 months after operation, while Schirmer results were $14.63,12.11$, and $14.14 \mathrm{~mm}$, respectively. In a similar study that enrolled the European population [22], the BUT values were 9.0, 10.9, and $11.6 \mathrm{~s}$ before operation and 1 and 3 months after SMILE, while Schirmer results were $17.5,16.6$, and $17.3 \mathrm{~mm}$ at aforementioned time points. These ocular surface parameters showed the same change tendencies as our study, and absolute values of tear secretion reduction were also similar. However, it appears that the tear film stability was worse after ICL surgeries in our study, for the decreasing extent was much more than that of SMILE surgeries and the recovery was slower as well. This also proved that ICL surgeries influence ocular surface functions more severely than SMILE, perhaps because the incisions were larger and deeper.

It is obvious in this study that the postoperative tear secretion decreased mildly and recovered to preoperative levels within 3 months, as in other similar ocular surface surgeries. Therefore, the occurrence or aggravation of the postoperative DE is not caused by the decrease in tear secretion, and the operations do not affect the tear secretion system.

In all postoperative $\mathrm{DE}$ and MG indicators, BUT value changed most violently, which decreased to one-third of the preoperative level at 1 month postoperatively, namely the stability of the tear film fell sharply and recovered slowly. The BUT value did not return to preoperative levels until the end of the follow-up period. It is well known that the tear film stability is closely related to the MG function. In this study, the MG-related parameters also presented a consistent trend with BUT values. Lid margin abnormalities and the quantity and quality of MG secre- 
tion changed obviously after surgeries. Meanwhile, the destruction of the ocular surface lipid layer was relatively severe, which was more likely to be the cause of postoperative DE. Our results are consistent with the reports of Han et al. [23], which analyzed preoperative and postoperative parameters of DE and MGD and found that the MGD changes significantly influenced the stability of tear film and caused the postoperative DE without altering the ocular surface structures.

In order to examine the effect of potential preoperative $\mathrm{DE}$ on postoperative $\mathrm{DE}$, we divided the patients into 2 groups according to the preoperative OSDI values: $\mathrm{DE}$ group (OSDI $>12$ ) and normal group (OSDI $\leq 12$ ). Patients in DE group showed more apparent subjective symptoms, whose OSDI values were about 4 times as those of the normal patients 1 month after operation. In addition, the tear secretion and tear film stability of patients in the DE group were worse and the differences between the 2 groups were significant not only before but also after ICL surgeries. Interestingly, the MG parameters of the 2 groups showed no obvious differences before operations but turned out huge differences after operations. For example, preoperative lid margin scores were similar in both groups, whereas the lid margin abnormalities scores of the DE group were almost twice those of the normal group 1 and 3 months after operation. So were the MG secretion quantity and quality scores. The aggravated differences after operations indicated that the ocular surface microenvironment was more vulnerable and more easily affected by the operation in DE group patients. Nevertheless, MG loss scores had no obvious changes in both groups, namely, though subjective symptoms and objective indexes had fluctuated sharply, the deep anatomy was not affected seriously or the influences had not yet appeared in 3-month follow-up period.

Our study found that tear secretion quantity and tear stability had the closest relationship with the subjective symptoms of DE, followed by MG secretion. Less Schirmer and BUT values, or the higher the meibum quality score, the more serious the DE symptoms. Therefore, it is important to complete the ocular surface detections, which could provide indicators to predict postoperative DE severity.

With these data, we are able to communicate in detail with patients and family members and help them prepare well. Moreover, perioperative drugs could be used for improving the lack of tear secretion or MGD, in order to reduce damage to the ocular surface microenvironment, relieve symptoms, or avoid deterioration, which play a positive role in the physiological and psychological health of patients.

Dry Eye after ICL Implantation
In conclusion, persistent or serious DE after ICL surgery was a common cause that affects the operation effects. Patients who had preoperative DE were the most likely to complain symptoms and their ocular surface microenvironments were more vulnerable to operations. Tear secretion quantity, tear film stability, and traits of MG secretions appeared to have very close relationship with the degree of postoperative DE. Surgeons should monitor these parameters before and after ICL surgeries and take measures to improve the satisfaction of the patients.

\section{Statement of Ethics}

The study was approved by the Cangzhou Central Hospital Ethics Committee and registered at the Chinese Clinical Trial Registry (ChiCTR-INR-16008896). These protocols followed the tenets of the Declaration of Helsinki, and written informed consent was obtained from all patients after they received a full explanation of the nature and possible consequences of the study.

\section{Conflict of Interest Statement}

The authors declare that there is not any conflicts of interest regarding the publication of this paper.

\section{Funding Sources}

No funding was achieved for this study.

\section{Author Contributions}

C.H. contributed to design of the study, acquisition of data, analysis and interpretation of data, and writing the article. N.G. participated in the recruitment of patients and acquisition of data. F.X. helped to recruit patients and contributed to conception and design of the study. F.Y. contributed to the resolution of major issues and modification of the manuscript and carried out all the operations. All authors read and agreed to the final manuscript.

References

1 Sanders DR, Schneider D, Martin R, Brown D, Dulaney D, Vukich J, et al. Toric Implantable Collamer Lens for moderate to high myopic astigmatism. Ophthalmology. 2007; 114(1):54-61.

2 Sanders DR, Doney K, Poco M. United States Food and Drug Administration clinical trial of the Implantable Collamer Lens (ICL) for moderate to high myopia: three-year followup. Ophthalmology. 2004;111(9):1683-92. 
3 Shimizu K, Kamiya K, Igarashi A, Shiratani T. Early clinical outcomes of implantation of posterior chamber phakic intraocular lens with a central hole (Hole ICL) for moderate to high myopia. Br J Ophthalmol. 2012;96(3): 409-12.

4 Lee J, Kim Y, Park S, Bae J, Lee S, Park Y, et al. Long-term clinical results of posterior chamber phakic intraocular lens implantation to correct myopia. Clin Experiment Ophthalmol. 2016;44(6):481-7.

5 Kamiya K, Shimizu K, Igarashi A, Kitazawa Y, Kojima T, Nakamura T, et al. Posterior chamber phakic intraocular lens implantation: comparative, multicentre study in 351 eyes with low-to-moderate or high myopia. $\mathrm{Br} \mathrm{J}$ Ophthalmol. 2018;102(2):177-81.

6 Inomata T, Iwagami M, Hiratsuka Y, Fujimoto K, Okumura Y, Shiang T, et al. Maximum blink interval is associated with tear film breakup time: a new simple, screening test for dry eye disease. Sci Rep. 2018;8(1):13443.

7 Cohen E, Spierer O. Dry eye post-laser-assisted in situ keratomileusis: major review and latest updates. J Ophthalmol. 2018;2018:1-9.

8 Iglesias E, Sajnani R, Levitt RC, Sarantopoulos $\mathrm{CD}$, Galor A. Epidemiology of persistent dry eye-like symptoms after cataract surgery. Cornea. 2018;37(7):893-8.

9 Acera A, Rocha G, Vecino E, Lema I, Durán JA. Inflammatory markers in the tears of patients with ocular surface disease. Ophthalmic Res. 2008;40(6):315-21.
10 Song P, Sun Z, Ren S, Yang K, Deng G, Zeng $\mathrm{Q}$, et al. Preoperative management of MGD alleviates the aggravation of MGD and dry eye induced by cataract surgery: a prospective, randomized clinical trial. BioMed Res Inter. 2019;2019:1-10.

11 Ding Y. The Prevalence of Demodex Folliculorum on the Eyelashes of Patients with Meibomian Gland Dysfunction. Master Paper. Dalian Medical University; 2014.

12 Tomlinson A, Bron AJ, Korb DR, Amano S, Paugh JR, Pearce EI, et al. The international workshop on meibomian gland dysfunction: report of the diagnosis subcommittee. Invest Ophthalmol Vis Sci. 2011;52(4):2006-49.

13 Arita R, Itoh K, Inoue K, Amano S. Noncontact infrared meibography to document agerelated changes of the meibomian glands in a normal population. Ophthalmology. 2008; 115(5):911-5.

14 Lane SS, DuBiner HB, Epstein RJ, Ernest PH, Greiner JV, Hardten DR, et al. A new system, the LipiFlow, for the treatment of meibomian gland dysfunction. Cornea. 2012;31(4):396404.

15 Bron AJ, Evans VE, Smith JA. Grading of corneal and conjunctival staining in the context of other dry eye tests. Cornea. 2003;22(7): 640-50.

16 Mian SI, Li AY, Dutta S, Musch DC, Shtein RM. Dry eyes and corneal sensation after laser in situ keratomileusis with femtosecond laser flap creation Effect of hinge position, hinge angle, and flap thickness. J Cataract Refract Surg. 2009;35(12):2092-8.
17 Kasetsuwan N, Satitpitakul V, Changul T, Jariyakosol S. Incidence and pattern of dry eye after cataract surgery. PLoS One. 2013;8(11): e78657.

18 Li XM, Hu L, Hu J, Wang W. Investigation of dry eye disease and analysis of the pathogenic factors in patients after cataract surgery. Cornea. 2007;26(9 Suppl 1):S16-20.

19 Choi YJ, Park SY, Jun I, Choi M, Seo KY, Kim EK, et al. Perioperative ocular parameters associated with persistent dry eye symptoms after cataract surgery. Cornea. 2018;37(6):7349.

20 Li M, Zhao J, Shen Y, Li T, He L, Xu H, et al Comparison of dry eye and corneal sensitivity between small incision lenticule extraction and femtosecond LASIK for myopia. PLoS One. 2013;8(10):e77797.

21 Xia L, Zhang J, Wu J, Yu K. Comparison of corneal biological healing after femtosecond LASIK and small incision lenticule extraction procedure. Curr Eye Res. 2016;41(9):1202-8.

22 Demirok A, Ozgurhan EB, Agca A, Kara N Bozkurt E, Cankaya KI, et al. Corneal sensation after corneal refractive surgery with small incision lenticule extraction. Optom Vis Sci. 2013;90(10):1040-7.

23 Han KE, Yoon SC, Ahn JM, Nam SM, Stulting $\mathrm{RD}$, Kim EK, et al. Evaluation of dry eye and meibomian gland dysfunction after cataract surgery. Am J Ophthalmol. 2014;157(6): 1144-50.e1. 